Original article

\title{
C-reactive protein mediates the association of liver fat and carotid intima-media thickness in healthy men and men with the metabolic syndrome and/or

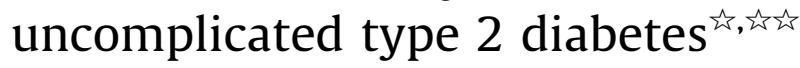

\author{
Nynke J. Van der Zijl ${ }^{\mathrm{a}, 1, *}$, Marcel H.A. Muskiet ${ }^{\mathrm{a}, 1}$, Maarten E. Tushuizen ${ }^{\mathrm{a}}$, Luuk-Jon Rijzewijk ${ }^{\mathrm{a}}$, \\ Roger K. Schindhelm ${ }^{\text {b }}$, Chantalle C.M. Moors ${ }^{c}$, Gijs H. Goossens ${ }^{c}$, Ellen E. Blaak ${ }^{c}$, \\ Petra J. Pouwels ${ }^{\mathrm{d}}$, Michaela Diamant ${ }^{\mathrm{a}}$ \\ ${ }^{a}$ Department of Endocrinology/Diabetes Centre, VU University Medical Centre, Amsterdam, The Netherlands \\ ${ }^{\mathrm{b}}$ Department of Clinical Chemistry, Isala Clinics, Zwolle, The Netherlands \\ ${ }^{\mathrm{c}}$ Department of Human Biology, School for Nutrition, Toxicology and Metabolism (NUTRIM), Maastricht University Medical Centre, Maastricht, The Netherlands \\ ${ }^{\mathrm{d}}$ Department of Physics \& Medical Technology, VU University Medical Centre, Amsterdam, The Netherlands
}

\section{A R T I C L E I N F O}

\section{Keywords:}

Type 2 diabetes

Metabolic syndrome

Liver fat

C-reactive protein

Carotid intima-media thickness

\begin{abstract}
A B S T R A C T
We investigated whether the relation between liver fat and subclinical atherosclerosis is mediated by low-grade systemic inflammation in type 2 diabetes (T2DM) and the metabolic syndrome (MetS).

In 16 T2DM, 36 MetS, and 21 age-matched healthy men, liver fat was quantified by ${ }^{1} \mathrm{H}-\mathrm{MRS}$. Carotid intima-media thickness (cIMT) was measured by ultrasound. Differences between controls, MetS and T2DM or between high versus low liver fat content were calculated. Furthermore, the association of liver fat content, cIMT and CRP was assessed.

Liver fat percentage was highest in T2DM, as compared to MetS and controls [17.5\% (8.0-27.6), 8.2\% (4.2-14.2) and 5.3\% (2.3-7.4), respectively; $P<0.001$ ]. Subjects with high-liver fat content had increased cIMT and CRP (all $P<0.02$ ). Liver fat content was positively associated with cIMT $(r=0.37 ; P=0.003)$. The association of liver fat content and cIMT disappeared after adjustment for CRP.

The association between liver fat content and subclinical atherosclerosis seems mediated by lowgrade inflammation, possibly due to increased hepatic production. Lowering of liver fat content may decrease CVD risk by concomitantly reducing the pro-inflammatory state in high-risk populations.
\end{abstract}

( 2010 Diabetes India. Published by Elsevier Ltd. All rights reserved.

\section{Introduction}

The incidence of non-alcoholic fatty liver disease (NAFLD) increases globally and, consequently, has gained increasing interest in recent years [1]. NAFLD is characterized by triglyceride accumulation in hepatocytes and includes a wide spectrum of liver pathology, ranging from benign liver steatosis to non-alcoholic steatohepatitis (NASH), the latter which may progress to cirrhosis [1]. NAFLD develops without a history of excessive alcohol consumption or positive tests for viral and auto-immune liver diseases [1,2]. NAFLD is highly prevalent in insulin resistant subjects

\footnotetext{
Grant support: This research received no specific grant from any funding agency in the public, commercial, or not-for-profit sectors.

Registration number: ISRCTN 427863362007.

* Corresponding author at: Diabetes Centre/Department of Internal Medicine, VU University Medical Centre, PO Box 7057, 1007 MB Amsterdam, The Netherlands. Tel.: +31 204442974; fax: +31204440502.

E-mail address: nj.vdzijl@vumc.nl (N.J. Van der Zijl).

${ }^{1}$ Both authors contributed equally.
}

such as individuals with the MetS and T2DM [3-6], who are additionally characterized by an increased cardiovascular disease (CVD) risk [7]. Indeed, several studies have demonstrated that NAFLD is associated with CVD [8], carotid artery atherosclerosis [911] and endothelial dysfunction [12], independent of classical risk factors such as insulin resistance and components of the MetS.

Although the mechanisms underlying the association of NAFLD and CVD still remain to be clarified, NAFLD is characterized by an increased pro-inflammatory state, including elevated plasma Creactive protein (CRP) levels [3,13]. CRP is marked as an independent predictor of future CVD events [14-16]. Therefore, this pro-inflammatory phenotype may underlie the association between liver fat and CVD risk. Carotid intima-media thickness (cIMT), assessed by ultrasound, is a validated parameter for subclinical atherosclerosis [17], predictive of both myocardial infarction and stroke [18]. cIMT has been related to the severity of liver damage in NAFLD, as measured by liver biopsy [19] and by ultrasound [11]. However, at present, the inter-relationship of liver fat accumulation, subclinical atherosclerosis and a pro-inflammatory state is still incompletely understood. 
We hypothesized that the association between liver fat content and subclinical atherosclerosis may be mediated by low-grade systemic inflammation, reflected by increased CRP concentrations. To this purpose, we measured liver fat by proton magnetic resonance spectroscopy $\left({ }^{1} \mathrm{H}-\mathrm{MRS}\right)[20]$, cIMT by ultrasound and plasma CRP concentrations in men with uncomplicated T2DM, the MetS and healthy age-matched controls.

\section{Materials and methods}

\subsection{Study population}

After written informed consent, 73 Caucasian males, aged 4065 years, with known T2DM $(n=16)$, MetS $(n=36)$ and healthy controls $(n=21)$ were studied. Diet, sulphonylurea and/or metformin were the only blood glucose-lowering treatments allowed in the T2DM group. Subjects with the MetS or T2DM were allowed to use low dose blood pressure lowering medication (angiotensin receptor antagonists, angiotensin converting enzyme inhibitors, calcium channel blockers or thiazide diuretics). Exclusion criteria comprised claustrophobia, smoking, excessive alcohol intake ( $>20$ units/week), history of hepatitis and/or pancreatitis, history of CVD, abnormal liver and renal function tests ( $>2$ times upper limits of normal), recent ( $<3$ months) changes in medication or weight $(\geq 5 \%)$ and any other medication. The study was approved by the local Ethics Committee and was performed in line with the Helsinki Declaration of human rights.

\subsection{Study protocol}

Following an overnight fast, the subjects arrived at the hospital clinical research unit in the morning for ultrasound examination of the common carotid artery and venous blood sampling. At a separate visit, liver fat content was measured in the fasting state using ${ }^{1} \mathrm{H}-\mathrm{MRS}$. All measurements were completed within 4 weeks after the screening visit.

\subsection{Laboratory analysis}

Baseline plasma glucose concentrations were determined by the hexokinase-method (Gluco-quant, Roche Diagnostics, Mannheim, Germany). Haemoglobin $\mathrm{A}_{1 \mathrm{c}}\left(\mathrm{HbA}_{1 \mathrm{c}}\right)$ was measured with cation exchange chromatography (Menarini Diagnostics, Florence, Italy). The local reference range for nondiabetic subjects is 4.3$6.1 \%$. Total cholesterol, high-density lipoprotein cholesterol (HDLC) and triglycerides (TG) were determined by enzymatic methods (Modular, Hitachi, Japan). Low-density lipoprotein cholesterol (LDL-C) was calculated with the Friedewald formula [21]. Insulin (reference cut-off value: $<20 \mu \mathrm{U} / \mathrm{mL}$ ) was determined by radioimmunoassay (Medgenix, Brussels, Belgium). Insulin resistance was evaluated by using the index HOMA-IR (homeostasis model assessment of insulin resistance) [HOMA-IR: fasting plasma glucose $(\mathrm{mmol} / \mathrm{L}) \times$ fasting serum insulin $(\mu \mathrm{U} / \mathrm{mL}) / 22.5]$ [22] CRP was determined by a highly sensitive, latex particle-enhanced immunoturbidimetric assay (reference range: $0.1-6.0 \mathrm{mg} / \mathrm{L}$; intraassay coefficient of variation (CV) was $4 \%$ and inter-assay CV was $6 \%)$.

\subsection{Diagnosis of the metabolic syndrome}

The MetS was diagnosed according to criteria of the International Diabetes Federation (IDF) [23]. To this end, central obesity (waist circumference $\geq 90 \mathrm{~cm}$ in men) and two or more of the following criteria should be present: (1) hypertriglyceridaemia ( $\geq 1.7 \mathrm{mmol} / \mathrm{L}$ ), or specific treatment for this lipid abnormality; (2) low HDL-C $(<0.9 \mathrm{mmol} / \mathrm{L}$ in men), or specific treatment for this lipid abnormality; (3) high blood pressure $(\geq 130 / 85 \mathrm{~mm} \mathrm{Hg})$, or treatment of previously diagnosed hypertension; (4) hyperglycaemia: fasting plasma glucose level $\geq 5.6 \mathrm{mmol} / \mathrm{L}$, and/or 2 -h postload plasma glucose level $\geq 7.8 \mathrm{mmol} / \mathrm{L}$. T2DM patients were not included in the MetS group.

\subsection{Assessment of common cIMT}

Ultrasonography scanning of the right common carotid artery was performed in order to measure cIMT. Measurements were performed by an experienced investigator employing a B-mode ultrasound imager with a 7.5 MHz linear-array transducer (Esaote, Maastricht, The Netherlands). Examination took place in the supine position, with the subjects' head turned slightly to the contralateral (left) side. When optimal longitudinal images were captured during the systole of a single heartbeat, they were stored for off-line analysis using the Wall Track System 2 software (Neurodata, Bilthoven, The Netherlands). Subsequently, the thickness of the intima-media complex (i.e. the distance between the edge of the first and second echogenic lines) was measured on the posterior (far) wall of the common carotid artery at about $10 \mathrm{~mm}$ proximal to the carotid bulb. For each subject, ultrasound data were obtained during three consecutive measurements, each comprising a 4-s period (approximately 4 heart beats). The mean cIMT was used in the analyses. A study of the intra-observer reproducibility of cIMT measurements in 10 healthy subjects revealed a CV of less than $2 \%$.

\subsection{Quantification of liver fat content}

Quantification of liver fat content was performed by ${ }^{1} \mathrm{H}-\mathrm{MRS}$ with the subject in the supine position using a 1.5-T whole-body magnetic resonance scanner (Sonata; Siemens, Erlangen, Germany), as previously described [20,24]. For the localization of the voxel of interest (VOI) both axial and coronal high resolution magnetic resonance imaging scans were used. A $8-15 \mathrm{~cm}^{3}$ VOI (voxel length ranging from 2.0 to $2.5 \mathrm{~cm}$ ) was placed at three positions in the liver (right anterior, right posterior, and medial or left anterior) avoiding vascular structures, intrahepatic bile ducts, subcutaneous fat tissue and the lateral margin of the liver. Spectral quantification was performed with LC Model (version 6.1) [25]. Triglyceride content was expressed as the ratio of the area under the methyl $(0.9 \mathrm{ppm})$ and methylene peaks (1.3 ppm) relative to the area under the water peak (4.65 ppm) [19]. The finally used triglyceride content was the mean of three VOIs. All measurements were analyzed by analysts unaware of the subjects' clinical data. High-liver fat content was defined as having a liver fat content $\geq 5.56 \%$, whereas subjects with liver fat content $<5.56 \%$ were designated as having low liver fat content [20]. The CV between two repeated assessments of liver fat content was $4.7 \%$ [24].

\subsection{Statistical analyses}

Results are presented as mean values \pm standard deviation (SD) or, in case of skewed distribution, as median values (interquartile range; IQR). Non-Gaussian distributed data, based on QQ-plots, were log-transformed to normality before analysis. Between-group differences, i.e. between controls, MetS and T2DM or between high versus low liver fat content, were calculated by analysis of variance (ANOVA) and Bonferroni post hoc analysis was used to correct for multiple comparisons. The associations between cIMT and selected cardiometabolic risk factors were assessed by univariate correlations. The role of CRP as a mediator in the associations between liver fat content and cIMT was assessed by multivariate regression analysis. In this 
Table 1

Clinical characteristics in males with T2DM, the MetS and healthy controls.

\begin{tabular}{|c|c|c|c|c|}
\hline & Controls $(n=21)$ & MetS $(n=36)$ & $\mathrm{T} 2 \mathrm{DM}(n=16)$ & $P$-Value \\
\hline Age (years) & $55.9 \pm 7.9$ & $56.6 \pm 6.9$ & $55.4 \pm 4.8$ & 0.84 \\
\hline Body mass index $\left(\mathrm{kg} / \mathrm{m}^{2}\right)$ & $27.7 \pm 3.2$ & $29.4 \pm 2.8$ & $31.7 \pm 4.2^{\ddagger}$ & 0.001 \\
\hline Waist circumference $(\mathrm{cm})$ & $101.6 \pm 9.0$ & $107.5 \pm 8.2^{*}$ & $110.8 \pm 9.7^{*}$ & 0.006 \\
\hline Systolic blood pressure (mmHg) & $126 \pm 11$ & $136 \pm 14^{*}$ & $135 \pm 13$ & 0.021 \\
\hline Diastolic blood pressure (mmHg) & $78 \pm 7$ & $86 \pm 7^{\ddagger}$ & $82 \pm 5$ & $<0.001$ \\
\hline Fasting plasma glucose $(\mathrm{mmol} / \mathrm{L})$ & $5.4(5.1-5.5)$ & $6.0(5.6-6.3)^{*}$ & $8.1(7.5-9.9)^{\ddagger . \S}$ & $<0.001$ \\
\hline $\operatorname{HbA} 1 \mathrm{c}(\%)$ & $5.5(5.4-5.8)$ & $5.9(5.6-6.1)$ & $6.9(6.5-8.0)^{\ddagger . \S}$ & $<0.001$ \\
\hline Plasma glucose $2-\mathrm{h}(\mathrm{mmol} / \mathrm{L})$ & $5.5(4.5-6.5)$ & $6.6(5.9-8.0)^{*}$ & $14.9(12.4-17.1)^{\ddagger . \S}$ & $<0.001$ \\
\hline Fasting insulin $(\mathrm{pmol} / \mathrm{L})$ & $34.7(23.1-62.6)$ & $55.5(43.1-81.8)^{\ddagger}$ & $77.2(42.2-82.2)^{\ddagger}$ & $<0.001$ \\
\hline HOMA-IR & $1.2(0.8-2.0)$ & $2.2(1.6-3.4)^{\ddagger}$ & $3.1(2.2-5.6)^{\ddagger . \dagger}$ & $<0.001$ \\
\hline Total cholesterol (mmol/L) & $5.2 \pm 0.8$ & $5.2 \pm 1.0$ & $5.0 \pm 0.6$ & 0.77 \\
\hline $\mathrm{HDL}-\mathrm{C}(\mathrm{mmol} / \mathrm{L})$ & $1.5 \pm 0.4$ & $1.1 \pm 0.3^{\ddagger}$ & $1.1 \pm 0.3^{\ddagger}$ & $<0.001$ \\
\hline $\mathrm{LDL}-\mathrm{C}(\mathrm{mmol} / \mathrm{L})$ & $3.2 \pm 0.7$ & $3.3 \pm 0.8$ & $3.1 \pm 0.6$ & 0.72 \\
\hline Triglycerides (mmol/L) & $1.0(0.8-1.1)$ & $1.7(1.2-2.3)^{\ddagger}$ & $1.8(1.3-2.8)^{\ddagger}$ & $<0.001$ \\
\hline Gamma-GT (U/L) & $21(16-30)$ & $33(23-42)^{*}$ & $33(27-42)^{*}$ & 0.001 \\
\hline $\operatorname{ALT}(\mathrm{U} / \mathrm{L})$ & $23(17-40)$ & $30(22-44)$ & $31(25-48)$ & 0.073 \\
\hline \multicolumn{5}{|l|}{ Medication } \\
\hline Blood pressure lowering (\%) & 0 & 18 & 15 & 0.17 \\
\hline Oral blood glucose lowering (\%) & 0 & 0 & 62 & $<0.001$ \\
\hline
\end{tabular}

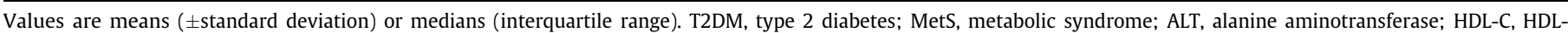
cholesterol; LDL-C, LDL-cholesterol; HOMA-IR, homeostasis model assessment of insulin resistance.

" $P<0.05$ compared to CO.

$¥ P<0.001$ compared to CO.

$\dagger P<0.05$ T2DM compared to MetS.

$\S P<0.001$ T2DM compared to MetS.

analysis, possible effect modification of the glucometabolic state (i.e. controls, MetS and T2DM) on the relationship between liver fat content and cIMT was evaluated by adding a composite interaction term. A $P$-value of $<0.05$ was considered statistically significant. For interaction-terms, a $P$-value of $<0.1$ was considered statistically significant. All statistical analyses were performed with SPSS version 15.0 (SPSS, Chicago, IL, USA).

\section{Results}

The baseline clinical and biochemical characteristics of the subjects are listed in Table 1. As expected, T2DM patients had the highest BMI, waist circumference, heart rate, HbA1c, fasting plasma glucose, 2-h plasma glucose, triglyceride concentrations, fasting plasma insulin concentrations, and HOMA-IR, whereas their HDL-C concentrations were lowest. Males with the MetS had the highest blood pressure levels.

Liver fat content gradually increased over the three groups from 5.3\% (2.3-7.4) (median (IQR)), 8.2\% (4.2-14.2) and 17.5\% (8.0-27.6), $P<0.001$ for controls, MetS, and T2DM, respectively. Mean $( \pm \mathrm{SD})$ cIMT was increased in MetS and T2DM compared to controls (controls: $0.64 \mathrm{~mm} \pm 0.14$, MetS: $0.71 \mathrm{~mm} \pm 0.16$ and T2DM: $0.69 \mathrm{~mm} \pm 0.12, P=0.035)$. CRP levels were highest in T2DM subjects (T2DM: $1.9 \mathrm{mg} / \mathrm{L}(0.9-3.0)$, MetS: $1.2 \mathrm{mg} / \mathrm{L}(0.9-$ 2.6) and controls: $0.9 \mathrm{mg} / \mathrm{L}(0.4-2.0), P=0.034)$. Of T2DM, $87 \%$ had high-liver fat content, as compared to $69 \%$ of MetS and $38 \%$ of controls $(P=0.005)$. When subjects were divided according to high versus low liver fat content, those with high-liver fat content had more CVD associated risk factors, including increased CRP and cIMT (Table 2).

\subsection{Associations of liver fat content, CRP and cIMT}

Positive correlations were found between liver fat content and cIMT $(r=0.28 ; P=0.03$; Fig. $1 \mathrm{~A})$ and liver fat content and CRP $(r=0.29 ; P=0.03$; Fig. 1B). In univariate analyses only age, systolicand diastolic blood pressure correlated significantly $(r=0.35$, $P=0.005 ; r=0.26, P=0.037 ; r=0.26, P=0.041$, respectively) with cIMT (Table 2 ).
To further analyse the independent relationship of liver fat content and subclinical atherosclerosis we used multivariate regression analyses (Table 3 ). Since the association between liver fat content and subclinical atherosclerosis did not differ in the three groups separately ( $P$-value of interaction term $>0.1$ ), effect modification was excluded and pooled analyses were performed. Model 1 describes the relation between liver fat content and cIMT. Glucometabolic state was added to the model to adjust for possible confounding (Model 2). When CRP was added to the model, the association between liver fat and cIMT was attenuated and lost significance, while a strong association between cIMT and CRP remained (Model 3). After adjustment for age and blood pressure the relation between CRP and cIMT sustained (Model 4).

Table 2

Biochemical and clinical differences in subjects with low- versus high-liver fat content.

\begin{tabular}{lccc}
\hline & $\begin{array}{l}\text { Liver fat } \\
\text { content } \\
<5.56 \%(n=26)\end{array}$ & $\begin{array}{l}\text { Liver fat } \\
\text { content } \\
\geq 5.56 \%(n=47)\end{array}$ & P-Value \\
& $55 \pm 7.9$ & $57 \pm 6.0$ & 0.43 \\
\hline Age (years) & $27 \pm 2.4$ & $31 \pm 3.4$ & $<0.001$ \\
Body mass index $\left(\mathrm{kg} / \mathrm{m}^{2}\right)$ & $110 \pm 8.8$ & $<0.001$ \\
Waist circumference $(\mathrm{cm})$ & $100.5 \pm 7$ & $6.1(5.5-7.5)$ & 0.027 \\
Fasting plasma & $5.6(5.4-6.1)$ & & \\
$\quad$ glucose (mmol/L) & $5.6(5.5-6.1)$ & $6.0(5.7-6.5)$ & 0.005 \\
HbA1c (\%) & $5.7(5.1-6.4)$ & $7.3(6.1-10.6$ & 0.001 \\
Plasma glucose & & & \\
2-h (mmol/L) & $22(17-28)$ & $33(26-45)$ & $<0.001$ \\
ALT (U/L) & $5.2 \pm 0.8$ & $5.2 \pm 0.9$ & 0.94 \\
Total cholesterol (mmol/L) & $1.4 \pm 0.4$ & $1.1 \pm 0.2$ & 0.001 \\
HDL-C (mmol/L) & $3.1 \pm 0.6$ & $3.2 \pm 0.8$ & 0.59 \\
LDL-C (mmol/L) & $1.1(0.9-1.5)$ & $1.6(1.1-2.3)$ & 0.007 \\
Triglycerides (mmol/L) & $32(24-49)$ & $67(47-85)$ & $<0.001$ \\
Fasting insulin (pmol/L) & $1.2(0.8-2.0)$ & $2.6(1.9-3.5)$ & $<0.001$ \\
HOMA-IR & $0.65 \pm 0.2$ & $0.74 \pm 0.1$ & 0.016 \\
cIMT (mm) & $0.9(0.4-1.8)$ & $1.7(0.9-2.9)$ & 0.001 \\
\hline CRP (mg/L) &
\end{tabular}

Values are means ( \pm standard deviation) or medians (interquartile range). ALT, alanine aminotransferase; HDL-C, high-density lipoprotein cholesterol; LDL-C, low-density lipoprotein cholesterol; HOMA-IR, homeostasis model assessment of insulin resistance; cIMT, carotid intima-media thickness; CRP, C-reactive protein. 
Table 3

Multivariate associations between liver fat content and cIMT.

\begin{tabular}{|c|c|c|c|c|c|}
\hline & $\Delta \mathrm{cIMT}(\mathrm{mm})(95 \% \mathrm{CI})$ & $P^{\mathrm{a}}$ & $r$ & $r^{2}$ & $P^{\mathrm{b}}$ \\
\hline $\begin{array}{l}\text { Model } 1 \\
\text { Liver fat content (\%) }\end{array}$ & $0.09(0.04-0.17)$ & 0.04 & 0.25 & 0.064 & 0.039 \\
\hline $\begin{array}{l}\text { Model } 2 \text { (Model } 1+\text { glucometabolic state) } \\
\text { Liver fat content }(\%) \\
\text { Glucometabolic state }\end{array}$ & $\begin{array}{c}0.09(0.004-0.18) \\
-0.05(-0.06 \text { to } 0.05)\end{array}$ & $\begin{array}{l}0.05 \\
0.86\end{array}$ & 0.25 & 0.064 & 0.089 \\
\hline $\begin{array}{l}\text { Model } 3 \text { (Model } 2+\text { CRP) } \\
\text { Liver fat content }(\%) \\
\text { Glucometabolic state } \\
\text { CRP }(\mathrm{mg} / \mathrm{L})\end{array}$ & $\begin{array}{l}0.04(-0.06 \text { to } 0.13) \\
0.009(-0.057 \text { to } 0.074) \\
0.12(-0.006 \text { to } 0.24)\end{array}$ & $\begin{array}{l}0.44 \\
0.79 \\
0.06\end{array}$ & 0.34 & 0.11 & 0.029 \\
\hline $\begin{array}{l}\text { Model } 4 \text { (Model } 3+\text { age and BP) } \\
\text { Liver fat content }(\%) \\
\text { Glucometabolic state } \\
\text { CRP (mg/L) } \\
\text { Age (years) } \\
\text { Systolic BP }(\mathrm{mmHg}) \\
\text { Diastolic BP }(\mathrm{mmHg})\end{array}$ & $\begin{array}{l}0.001(-0.09 \text { to } 0.09) \\
-0.014(-0.075 \text { to } 0.047) \\
0.13(0.03-0.24) \\
0.009(0.004-0.015) \\
0.001(-0.002 \text { to } 0.005) \\
0.004(-0.003 \text { to } 0.01)\end{array}$ & $\begin{array}{l}0.97 \\
0.64 \\
0.04 \\
0.015 \\
0.005 \\
0.011\end{array}$ & 0.57 & 0.32 & $<0.001$ \\
\hline
\end{tabular}

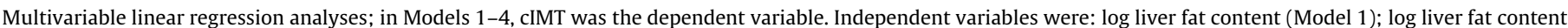

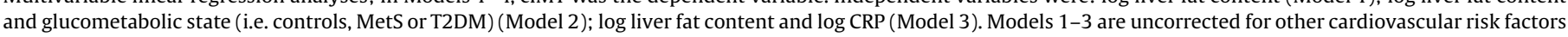

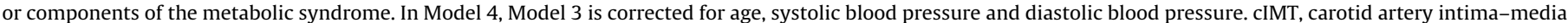
thickness; CI, confidence interval; CRP, C-reactive protein; BMI, body mass index; BP, blood pressure.

a Level of significance for the association between cIMT and the separate components of the model.

b Level of significance of the model.
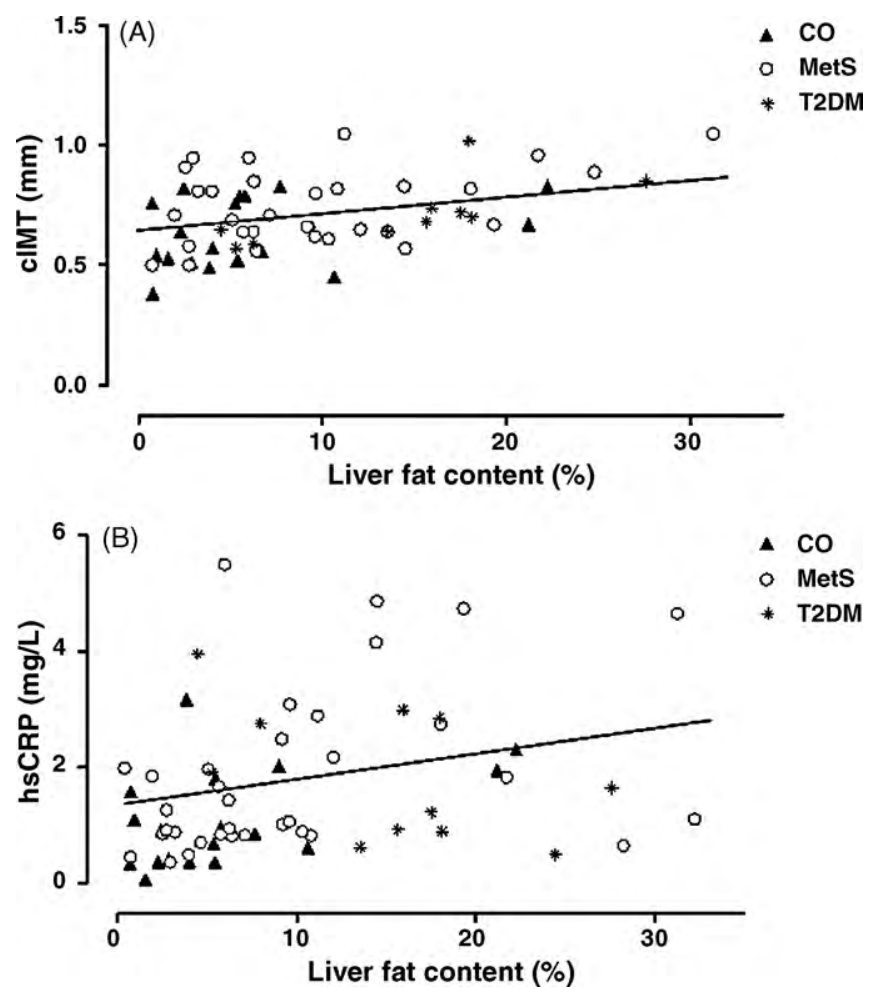

Fig. 1. Scatterplots showing the relationship of liver fat content and carotid artery intima-media thickness (CIMT) (A) and liver fat content and plasma C-reactive protein (CRP) concentrations (B) in males with type 2 diabetes (asterisk), the MetS (dot) and healthy controls (triangle).

\section{Discussion}

The present study demonstrates that subjects with high versus low liver fat content had higher CRP concentrations and more marked subclinical atherosclerosis, represented by an increased cIMT, irrespective of their glucometabolic differences. Furthermore, the positive association between liver fat content and carotid artery subclinical atherosclerosis disappeared after adjustment for plasma CRP concentration, suggesting that this circulating proinflammatory marker, that is largely derived from the liver, mediates the relation between the fatty liver and subclinical atherosclerosis in T2DM patients, subjects with the MetS and healthy controls.

The relation between NAFLD and markers of CVD is well established [9-12,19]. However, the mechanisms by which increased liver fat content could accelerate atherogenesis are not conclusive. The association between NAFLD and atherosclerosis may reflect the overall atherogenic condition pertinent to insulin resistant states such as the MetS and T2DM [1,7]. Insulin resistance results in decreased inhibition of hepatic glucose production, increased hepatic output of TG-rich lipoproteins but also decreased glucose utilization and lipolysis [3], leading to hyperglycaemia [26], hypertriglyceridaemia and increased circulating non-esterified fatty acids (NEFA) [27]. The increased lipid availability in the liver provides an atherogenic dyslipidaemic milieu with increased rates of hepatic triglyceride synthesis and VLDL particle production resulting in low HDL-cholesterol and increased small dense LDL particles [26-28]. In our study, differences in lipid profile could not explain the increase in subclinical atherosclerosis in subjects with high-liver fat content as compared tot subjects with low liver fat content.

Increased fluxes of NEFA, in addition to dietary chylomicrons and de novo intrahepatic lipogenesis, the latter driven by abundant substrate supply in the presence of hyperglycaemia, will result in intrahepatic triglyceride and lipid accumulation [1-3]. Besides, when NEFA exceeds oxidative capacity, this will lead to the formation of toxic intermediates, and ultimately to mitochondrial dysfunction and increased oxidative stress in NAFLD, which in turn results in cell damage and increased release of inflammatory cytokines, e.g. CRP [29]. Additionally, increased production of adipocytokines, including tumor necrosis factor-alpha and interleukin-6, by adipose tissue enhances the production of proinflammatory cytokines in the fatty liver. The ensuing systemic inflammation may play a key role in the increased CVD risk in subjects with NAFLD [29-31]. In our study liver fat content, gradually increased with deterioration of glucometabolic variables, regarded as MetS and T2DM, and this was paralleled by elevation of CRP. Furthermore, multivariate analyses outlined the role of CRP in the relationship between liver fat content and 
subclinical atherosclerosis. Although no causal relationship can be derived from our cross-sectional data, we may hypothesize that the increased CRP levels, possibly due to increased hepatic production, can directly or indirectly mediate the relationship between NAFLD and CVD.

Certain limitations of our study should be acknowledged, such as the cross-sectional design that precludes conclusions regarding causality of the found associations. Secondly, the use of HOMA-IR instead of an euglycaemic-hyperinsulinaemic clamp to estimate insulin resistance. HOMA-IR may be less reliable in subjects with fatty liver, since liver fat impairs insulin clearance [32], and in subjects with T2DM, where elevated fasting plasma glucose may drive the HOMA-IR value. Finally, although an excellent correlation exists between ${ }^{1} \mathrm{H}$-MRS-measured liver fat content and the fat content quantified in liver biopsy specimen [20], the gold-standard biopsy technique may provide additional information regarding the presence of an inflammatory component or the severity of NASH.

In conclusion, we have demonstrated that liver fat content is associated with subclinical atherosclerosis, and that this association may be mediated by CRP, a pro-inflammatory marker that is known to be largely derived from the liver. A clinical diagnosis of NAFLD or an expected high-liver fat content, as concurs often with the MetS/T2DM phenotype, should prompt clinicians to even more aggressively treat the associated CVD risk factors to lower CVD risk.

\section{References}

[1] Angulo P. Nonalcoholic fatty liver disease. N Engl J Med 2002;346(16):122131.

[2] Brunt EM. Nonalcoholic steatohepatitis: definition and pathology. Semin Liver Dis 2001;21(1):3-16.

[3] Kotronen A, Yki-Järvinen H. Fatty liver: a novel component of the metabolic syndrome. Arterioscler Thromb Vasc Biol 2008;28(1):27-38.

[4] Schindhelm RK, Diamant M. Heine RJ. Nonalcoholic fatty liver disease and cardiovascular disease risk. Curr Diab Rep 2007;7(3):181-7.

[5] Cortez-Pinto H, Camilo ME, Baptista A, De Oliveira AG, De Moura MC. Nonalcoholic fatty liver: another feature of the metabolic syndrome? Clin Nutr 1999;18(6):353-8.

[6] Marchesini G, Brizi M, Bianchi G, Tomassetti S, Bugianesi E, Lenzi M, et al. Nonalcoholic fatty liver disease: a feature of the metabolic syndrome. Diabetes 2001;50(8):1844-50.

[7] Eckel RH, Grundy SM, Zimmet PZ. The metabolic syndrome. Lancet 2005; 365(9468):1415-28.

[8] Targher G, Bertolini L, Poli F, Rodella S, Scala L, Tessari R, et al. Nonalcoholic fatty liver disease and risk of future cardiovascular events among type 2 diabetic patients. Diabetes 2005;54(12):3541-6.

[9] Brea A, Mosquera D, Martín E, Arizti A, Cordero JL, Ros E. Nonalcoholic fatty liver disease is associated with carotid atherosclerosis: a case-control study. Arterioscler Thromb Vasc Biol 2005;25(5):1045-50.

[10] Fracanzani AL, Burdick L, Raselli S, Pedotti P, Grigore L, Santorelli G, et al. Carotid artery intima-media thickness in nonalcoholic fatty liver disease. Am J Med 2008;121(1):72-8.

[11] Targher G, Bertolini L, Padovani R, Poli F, Scala L, Zenari L, et al. Non-alcoholic fatty liver disease is associated with carotid artery wall thickness in dietcontrolled type 2 diabetic patients. J Endocrinol Invest 2006;29(1):55-60.
[12] Schindhelm RK, Diamant M, Bakker SJ, van Dijk RA, Scheffer PG, Teerlink T, et al. Liver alanine aminotransferase, insulin resistance and endothelial dysfunction in normotriglyceridaemic subjects with type 2 diabetes mellitus. Eur J Clin Invest 2005;35(6):369-74.

[13] Targher G, Bertolini L, Rodella S, Lippi G, Franchini M, Zoppini G, et al. NASH predicts plasma inflammatory biomarkers independently of visceral fat in men. Obesity (Silver Spring) 2008;16(June (6)):1394-9.

[14] Ridker PM. C-reactive protein and the prediction of cardiovascular events among those at intermediate risk: moving an inflammatory hypothesis toward consensus. J Am Coll Cardiol 2007;49(21):2129-38.

[15] Sander D, Schulze-Horn C, Bickel H, Gnahn H, Bartels E, Conrad B. Combined effects of hemoglobin A1c and C-reactive protein on the progression of subclinical carotid atherosclerosis: the INVADE study. Stroke 2006;37(2):351-7.

[16] Karakas M, Koenig W. CRP in cardiovascular disease. Herz 2009;34(8):607-13.

[17] O'Leary DH, Polak JF. Intima-media thickness: a tool for atherosclerosis imaging and event prediction. Am J Cardiol 2002;21(90 (10C)):18L-21L.

[18] O'Leary DH, Polak JF, Kronmal RA, Manolio TA, Burke GL, Wolfson Jr SK. Carotid-artery intima and media thickness as a risk factor for myocardial infarction and stroke in older adults. Cardiovascular Health Study Collaborative Research Group. N Engl J Med 1999;340(1):14-22.

[19] Targher G, Bertolini L, Padovani R, Rodella S, Zoppini G, Zenari L, et al. Relations between carotid artery wall thickness and liver histology in subjects with nonalcoholic fatty liver disease. Diabetes Care 2006;29(6):1325-30.

[20] Szczepaniak LS, Nurenberg P, Leonard D, Browning JD, Reingold JS, Grundy S, et al. Magnetic resonance spectroscopy to measure hepatic triglyceride content: prevalence of hepatic steatosis in the general population. Am J Physiol Endocrinol Metab 2005;288(2):E462-8.

[21] Friedewald WT, Levy RI, Fredrickson DS. Estimation of the concentration of low-density lipoprotein cholesterol in plasma, without use of the preparative ultracentrifuge. Clin Chem 1972;18(6):499-502.

[22] Matthews DR, Hosker JP, Rudenski AS, Naylor BA, Treacher DF, Turner RC. Homeostasis model assessment: insulin resistance and beta-cell function from fasting plasma glucose and insulin concentrations in man. Diabetologia 1985;28(7):412-9.

[23] Alberti KG, Zimmet P, Shaw J. Metabolic syndrome-a new worldwide definition: a consensus statement from the International Diabetes Federation. Diabetic Med 2006;23:469-80.

[24] Tushuizen ME, Bunck MC, Pouwels PJ, Bontemps S, Mari A, Diamant M. Lack of association of liver fat with model parameters of beta-cell function in men with impaired glucose tolerance and type 2 diabetes. Eur J Endocrinol 2008;159:251-7.

[25] Provencher SW. Estimation of metabolite concentrations from localized in vivo proton NMR spectra. Magn Reson Med 1993;30(6):672-9.

[26] Seppala-Lindroos A, Vehkavaara S, Hakkinen AM, Goto T, Westerbacka J, Sovijarvi A, et al. Fat accumulation in the liver is associated with defects in insulin suppression of glucose production and serum free fatty acids independent of obesity in normal men. J Clin Endocrinol Metab 2002;87:3023-8.

[27] Toledo FG, Sniderman AD, Kelley DE. Influence of hepatic steatosis (fatty liver) on severity and composition of dyslipidaemia in type 2 diabetes. Diabetes Care 2006;29:1845-50.

[28] Adiels M, Taskinen MR, Borén J. Fatty liver, insulin resistance, and dyslipidaemia. Curr Diab Rep 2008;8:60-4.

[29] Polyzos SA, Kountouras J, Zavos C. Nonalcoholic fatty liver disease: the pathogenetic roles of insulin resistance and adipocytokines. Curr Mol Med 2009;9(3):299-314.

[30] Yudkin JS, Stehouwer CD, Emeis JJ, Coppack SW. C-reactive protein in healthy subjects: associations with obesity, insulin resistance, and endothelial dysfunction: a potential role for cytokines originating from adipose tissue? Arterioscler Thromb Vasc Biol 1999;19(4):972-8.

[31] Festa A, D’Agostino Jr R, Howard G, Mykkänen L, Tracy RP, Haffner SM. Chronic subclinical inflammation as part of the insulin resistance syndrome: the Insulin Resistance Atherosclerosis Study (IRAS). Circulation 2000;102(July (1)):42-7.

[32] Kotronen A, Vehkavaara S, Seppälä-Lindroos A, Bergholm R, Yki-Järvinen H. Effect of liver fat on insulin clearance. Am J Physiol Endocrinol Metab 2007;293(6):E1709-15. 